\title{
Functional Organization of a Schwann Cell Enhancer
}

\author{
Eric Denarier, ${ }^{1}$ Reza Forghani, ${ }^{1}$ Hooman F. Farhadi, ${ }^{1}$ Samar Dib, ${ }^{1}$ Nancy Dionne, ${ }^{1}$ Hana C. Friedman, ${ }^{1}$ Pierre Lepage, ${ }^{2}$ \\ Thomas J. Hudson, ${ }^{2}$ Régen Drouin, ${ }^{3}$ and Alan Peterson ${ }^{1}$ \\ ${ }^{1}$ Laboratory of Developmental Biology, Molecular Oncology Group H-5, McGill University Health Centre, Montreal, Quebec, Canada H3A 1A1, ${ }^{2}$ McGill \\ University and Genome Quebec Innovation Centre, Montreal, Quebec, Canada H3A 1A4, and ${ }^{3}$ Service de Génétique Médicale, Département de Pédiatrie, \\ Centre Hospitalier Universitaire de Sherbrooke, Hôpital Fleurimont, Sherbrooke, Québec, Canada J1H 5N4
}

Myelin basic protein (MBP) gene expression is conferred in oligodendrocytes and Schwann cells by different upstream enhancers. In Schwann cells, expression is controlled by a $422 \mathrm{bp}$ enhancer lying $-9 \mathrm{~kb}$ from the gene. We show here that it contains 22 mammalian conserved motifs $\geq 6 \mathrm{bp}$. To investigate their functional significance, different combinations of wild-type or mutated motifs were introduced into reporter constructs that were inserted in single copy at a common hypoxanthine phosphoribosyltransferase docking site in embryonic stem cells. Lines of transgenic mice were derived, and the subsequent qualitative and quantitative expression phenotypes were compared at different stages of maturation. In the enhancer core, seven contiguous motifs cooperate to confer Schwann cell specificity while different combinations of flanking motifs engage, at different stages of Schwann cell maturation, to modulate expression level. Mutation of a Krox-20 binding site reduces the level of reporter expression, whereas mutation of a potential Sox element silences reporter expression. This potential Sox motif was also found conserved in other Schwann cell enhancers, suggesting that it contributes widely to regulatory function. These results demonstrate a close relationship between phylogenetic footprints and regulatory function and suggest a general model of enhancer organization. Finally, this investigation demonstrates that in vivo functional analysis, supported by controlled transgenesis, can be a robust complement to molecular and bioinformatics approaches to regulatory mechanisms.

Key words: myelin basic protein; Schwann cell; enhancer; phylogenetic footprint; transgenesis; HPRT

\section{Introduction}

Myelin is elaborated in the peripheral nervous system (PNS) and the CNS by Schwann cells and oligodendrocytes, respectively. Myelin basic protein (MBP) belongs to a family of coregulated genes that participates in the formation of myelin in both cell types, and several upstream regulatory modules variously contribute to its regulation in the two lineages (Farhadi et al., 2003) (Fig. 1A). Mod1 and Mod2, located in the proximal 5' flanking sequence, drive expression in myelinating oligodendrocytes, whereas Mod 3, located further upstream, confers expression in mature oligodendrocytes. Expression in Schwann cells is governed by Mod4, located in the mouse at $-9 \mathrm{~kb}$ (Forghani et al., 2001).

To locate the regulatory sequences controlling genes expressed in Schwann cells, previous investigations exploited reporter constructs containing specific promoter or enhancer se-

\footnotetext{
Received June 23, 2005; revised Sept. 15, 2005; accepted 0ct. 22, 2005.

This work was supported by grants from the Canadian Institutes of Health Research (CIHR), The Canadian Multiple Sclerosis Foundation, the Canadian Genetic Diseases Network, Genome Canada, and Genome Quebec. E.D. was supported by Commissariat à l'Energie Atomique, France, and Génome Québec. Doctoral fellowships were provided to R.F., H.F., S.D., and N.D. by CIHR, McGill University Health Center, and Multiple Sclerosis Society. T.J.H. is a recipient of an Investigator Award from CIHR and a Clinician-Scientist Award in Translational Research from the Burroughs Wellcome Fund. R.D. holds the "Canada Research Chair in Genetics, Mutagenesis, and Cancer." We thank P. Charnay for providing the pET-Krox plasmid. We are grateful to M. Beaudoin, F. Bourdeau, N. Dallaire, C. Dy, T. Fernandez, H. Furtenbacher, I. Paradis, I. Tretjakoff, and P. Valera for excellent technical support.

Correspondence should be addressed to Alan Peterson, Laboratory of Developmental Biology, Molecular Oncology Group H-5, McGill University Health Centre, 687 Pine Avenue West, Montreal, Quebec, Canada H3A 1A1. E-mail: alan@devbiol2.molonc.mcgill.ca.

DOI:10.1523/JNEUROSCI.2596-05.2005

Copyright $\odot 2005$ Society for Neuroscience $\quad 0270-6474 / 05 / 2511210-08 \$ 15.00 / 0$
}

quences. Regulatory sequences from P0 (Feltri et al., 1999), peripheral myelin protein 22 (PMP22) (Maier et al., 2003), and the transcription factors Oct-6 (Mandemakers et al., 2000) and Krox-20 (Ghislain et al., 2002) have been evaluated extensively in transgenic mice. However, the precise location, identity, and functional relevance of individual transcription factor binding elements are yet to be defined for any such locus.

Several transcription factors are known components of the mechanism engaged to regulate Schwann cell differentiation. In the absence of the zinc finger transcription factor Krox-20 or the POU-domain transcription factor Oct-6, Schwann cells arrest at the promyelinating stage (Topilko et al., 1994; Bermingham et al., 1996). In Oct-6 mutants, myelination resumes after a 2 week pause, whereas in Krox-20 mutants, this block is insurmountable. Sox-10, known to cooperate with Oct-6 (Kuhlbrodt et al., 1998) and Krox-20 (Bondurand et al., 2001), is also required for myelin formation (Britsch et al., 2001). Thus, differentiation of the Schwann cell lineage is controlled through a regulatory network of transcription factors acting at multiple stages of Schwann cell maturation. Evidence from in vitro studies suggests that these same factors may regulate individual myelin genes at later stages of maturation (Peirano et al., 2000; Musso et al., 2001).

Here, we attempted to refine the localization of functional elements in the MBP Mod4 Schwann cell enhancer by extending comparative analysis to additional species. We show, using a controlled strategy of transgenesis (Bronson et al., 1996), that phylogenetic footprints within this enhancer are functional regulatory elements. Additionally, functional analysis led to a model of enhancer structure in which targeting elements, clustered in the 


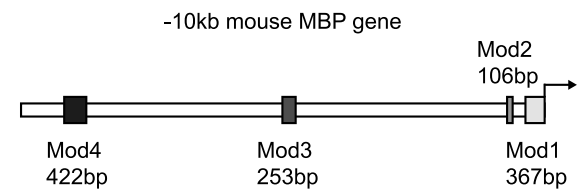

B

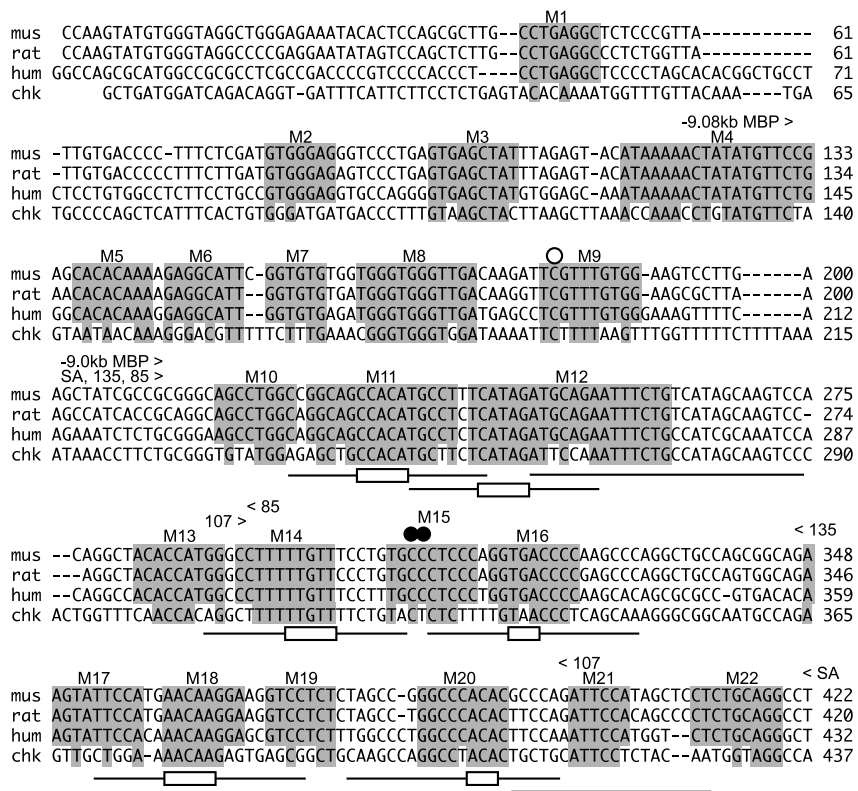

Figure 1. $\quad A$, Schematic representation of the mouse MBP gene $5^{\prime}$ flanking region showing the four conserved noncoding modules. $\boldsymbol{B}$, Sequence alignments of Mod 4 from four different species. Twenty-two motifs of at least $6 \mathrm{bp}$ are conserved in mammals. Motifs are highlighted and designated M1-M22. In vivo footprint analysis revealed a protected guanine (open circle over the sequence) and hypersensitive sites (filled circles). The Mod4 sequences evaluated for enhancer activity in reporter constructs are delineated by arrowheads over the sequence. Oligonucleotides used in EMSA (M11-M21) are indicated by lines under the sequence, and the nucleotides substituted in motif mutations are delineated by rectangles. mus, Mouse; hum, human; chk, chicken.

core, combine with enhancing activity contributed from multiple flanking motifs to confer fine control over cell specificity and expression levels. Notable differences in the enhancing elements engaged during myelin elaboration and maintenance demonstrate that the MBP expression program is controlled by different transcription factor repertoires at different stages of development. By specific mutation, we show that Krox-20 and a motif with potential Sox10 binding activity play major roles in enhancer function. Additionally, the Sox motif is conserved in other enhancer sequences active in myelinating Schwann cells, suggesting that it contributes widely to myelin gene expression in Schwann cells.

\section{Materials and Methods}

Generation of constructs for hypoxanthine phosphoribosyltransferase docked transgenesis. The MBP $-9.5 \mathrm{~kb}$ and $-9.0 \mathrm{~kb}$ constructs have been described previously (Farhadi et al., 2003). The construct $-9.08 \mathrm{~kb}$ was generated by adding an $80 \mathrm{bp}$ PCR product to the SacII site $5^{\prime}$ of clone $-9.0 \mathrm{~kb}$. To clone Mod4 subfragments, we first generated an hsp-LacZ Entry vector in which hsp-LacZ is cloned into an EcoRV site of the pENTRIA vector (Invitrogen, San Diego, CA). Mod4 subdomains were ligated upstream of the hsp promoter in reverse orientation relative to endogenous MBP. Entry vectors were used for in vitro recombination into the hypoxanthine phosphoribosyltransferase (HPRT) destination vector that includes homology arms for the HPRT locus using the LR clonase reaction kit (Invitrogen). The final destination vector was amplified, sequenced across the insert, and linearized by AgeI. Forty micrograms of targeting construct were used to transfect ES cells. Individual motif mutations were generated by a two-step PCR introducing a TTGTT $\rightarrow$ CGAGC substitution in 135-M14mut, TGA $\rightarrow$ ATT in 135M16mut, ACAA $\rightarrow$ CCGC in SA-M18mut, and ACA $\rightarrow$ GTT in SA-M20mut.

Derivation of transgenic mice. Transgenic animals bearing constructs in the HPRT docking site were generated by transfection of destination constructs bearing the HPRT targeting cassette into BK4 (for SA) or BPES5 cells, a laboratory-derived heterozygous cell line. Homologous recombination simultaneously restores the deleted HPRT locus in cells and inserts a single copy of the reporter construct into the HPRT 5' flanking region. Restoration of HPRT expression confers resistance to hypoxanthine-aminopterin-thymine selection, thus permitting positive selection for clones derived from the desired homologous recombination event. After selection and PCR screening, ES clones were used to inject blastocysts. DNAs prepared from chimeras or germline females were analyzed by Southern blot to check for single integration.

Remyelination experiments. To induce peripheral nerve regeneration, 2-month-old male mice were anesthetized, and unilateral sciatic nerve crushes were performed as described previously (Farhadi et al., 2003). The injury site was marked with India ink. After 1 or 2 weeks of recovery, injured and contralateral control nerves were recovered and dissected into similar proximal and distal segments. $\beta$-Galactosidase activity was measured in distal segments and compared with expression levels in uninjured contralateral samples.

Histochemical detection of $\beta$-galactosidase activity. Histochemical staining was performed as described previously (Forghani et al., 2001).

Quantitation of $\beta$-galactosidase activity. Sciatic nerves were dissected from back-cross two to three C57BL/6 male mice and snap frozen in liquid nitrogen. Forty-eight samples were homogenized using a mixer mill (Qiagen, Hilden, Germany) with a 2 min burst in $250 \mu$ l of lysis buffer. Total protein concentrations were measured for all extracts in triplicate by the Bradford procedure (Bio-Rad, Hercules, CA) using a BSA standard curve. $\beta$-Galactosidase activity was detected using Galacto-Star chemiluminescent assay system (Applied Biosystems, Foster City, CA). Standard curves were generated with serial dilutions of $\beta$-galactosidase (Roche, Welwyn Garden City, UK) in duplicate.

Quantification of MBP RNA. Sciatic nerves were dissected from anesthetized mice and snap frozen in liquid nitrogen. RNA was prepared from pools of four nerves [or eight nerves from postnatal day 2 (P2) and P4 mice] with the Qiagen RNAeasy Lipid mini kit according to the protocol of the manufacturer. The RNA was reverse transcribed using Superscript II (Invitrogen) and random hexamers according to the protocol of the manufacturer. Quantitative PCR was performed using the Roche LightCycler FastStart DNA Master plus SYBR Green I kit. The cDNA was diluted $1 / 10$ and $1 / 20$ and then measured using $2 \mu \mathrm{l}$ in a $20 \mu \mathrm{l}$ reaction. For each time point, three pools of nerves were measured and averaged. MBP cDNA was amplified with the following primers: CGAGAACTACCCATTATGGCTCCC and TGGAGGTGGTGTTCGAGGTGTC. Glyceraldehyde-3-phosphate dehydrogenase (GAPDH) was amplified with the following primers: ACCACAGTCCATGCCATCAC and TCCACCACCCTGTTGCTGTA. Results are expressed as MBP mRNA moles/GAPDH mRNA moles.

In vivo footprinting. Peripheral nerves from wild-type or Trembler mice, both on a C57BL/6 background, were analyzed. Trembler mice were evaluated here, because the chronic demyelination/remyelination they experience leads to an enrichment of myelinating Schwann cells in their peripheral nerves. Sciatic nerves were obtained from $\mathrm{P} 4-\mathrm{P} 7$ normal or adult Trembler mice and treated with dimethylsulfate (DMS), and their DNA was prepared for Maxam and Gilbert sequencing. Mice were killed, and their sciatic nerves were exposed. Each nerve was immersed in situ in Ringer's solution containing 0.5\% DMS for $6 \mathrm{~min}$ and then washed in cold $\mathrm{Ca}^{2+}$ - and $\mathrm{Mg}^{2+}$-free PBS for 1-5 min and then transferred to buffer $\mathrm{B}+\mathrm{C}(\sim 20$ nerves/milliliter). DNA was prepared and treated as described previously (Drouin et al., 2001). The sequence ( 800 bp) surrounding Mod4 was analyzed on both strands using different primer sets. As sequence marker, genomic DNA from the liver was pre- 
pared for sequencing. Methylated guanines from DMS exposures and chemically damaged DNA were converted into strand breaks by hot piperidine treatment. Strand break frequencies were estimated on an alkaline agarose gel (Drouin et al., 1996). Only consistent band intensity differences between in vivo and in vitro samples in two different experiments were scored as footprints.

Sequence analysis. The rat Mod4 sequence was obtained through the rat genome sequence (available at http://genome.ucsc.edu/). An MBP containing bacterial artificial chromosome (BAC) from the genomic Chicken BAC library (BACPAC resource; Oakland Research Institute, Oakland, CA) was identified using a PCR probe for chicken MBP exon 1. Positive clones were identified and amplified. DNA was sheared and ligated to pBluescript vectors. Sequences were aligned by ClustalW using MacVector 7.0 software. VISTA plot of M4 sequence was created using VISTA server (http://gsd.lbl.gov/vista/).

Electrophoretic mobility shift assays. Oligonucleotide probes were produced by annealing of the complementary strand and filling the two overhangs with $\left[\alpha-{ }^{32} \mathrm{P}\right] \mathrm{dCTP}$ and Klenow: M11 CTAGCCGGCAGCCACATGCCTTTC, M11mut CTAGCCGGCAGAATTCTGCCTTTC, M12-5' CTAGTGCCTTTCATAGATGCAGAA, M12-5'mut CTAGTGCCTTTGAATTCTGCAGAA, M14 CTAGGGGCCTTTTTGTTTCCTGTG, M14mut CTAGGGGCCTTTCGAGCTCCTGTG, M16 CTAGTCCCAGGTGACCCCAAGCCC,M16mutCTAGTCCCAGGATTCCCCAAGCCC, M20 CTAGTAGCCGGGCCCACACGCCCA, and KroxCTAGGTTGTACGCGGGGGCGGTTAGT. Preparation of sciatic nerve extracts was as follows: 150 sciatic nerves from P10 mice were dissected, extracts were prepared, and electrophoretic mobility shift assay (EMSA) reactions $(30 \mu \mathrm{l})$ were performed as described previously (Forghani et al., 1999). Bacterially expressed Krox was produced from pET-Krox plasmid as described previously (Nardelli et al., 1992). For supershift, $2.5 \mu \mathrm{g}$ of antibody (anti-Krox-20 Covance \#PRB-236P, Covance, Princeton, NJ; Sp1 Upstate \#07-124, Upstate Biotechnology, Lake Placid, NY) was added to the reaction mix before addition of the probe and preincubated for 15 min with the extracts.

\section{Results}

Interspecies sequence comparisons reveal conserved motifs within Mod4

The MBP 5' flanking sequence contains four noncoding sequences (Mod1 to Mod4), each with human/mouse conservation extending over $100 \mathrm{bp}$ at $75 \%$ or greater identity (Farhadi et al., 2003). Mod4 lies $9 \mathrm{~kb}$ upstream of the MBP start site and corresponds to a $422 \mathrm{bp}$ region of conservation with Schwann cell enhancer activity. To reveal motifs that may correspond to functional transcription factor binding sites, we extended the Mod 4 sequence comparison to rat and chicken. Mouse/rat, mouse/ human, and mouse/chicken comparisons yield sequence identities of 91,76 , and $56 \%$, respectively. Twenty-two motifs of at least $6 \mathrm{bp}$ (M1 to M22) are conserved in many mammalian species and, of these, seven are invariant in the more distantly related chicken species (Fig. 1).

\section{In vivo footprinting reveals protein/DNA interactions in the core Mod4 sequence}

To locate the regions of DNA-transcription factor interaction in the Mod4 region, we screened an 800 bp Mod4-containing sequence using the ligation-mediated PCR-based in vivo footprinting approach. DNA was derived from sciatic nerve samples from normal mice. To potentially enrich for the population of Schwann cells actively elaborating myelin, additional samples were prepared from Trembler mice. Sciatic nerves were pretreated with dimethylsulfate, and DNA was purified and subsequently analyzed for footprints. Within the $800 \mathrm{bp}$ region screened in this analysis, three footprints were detected, all located in the core of the conserved Mod4 sequence. One is a protected guanine residue in motif 9 , and two are hypersensitive sites
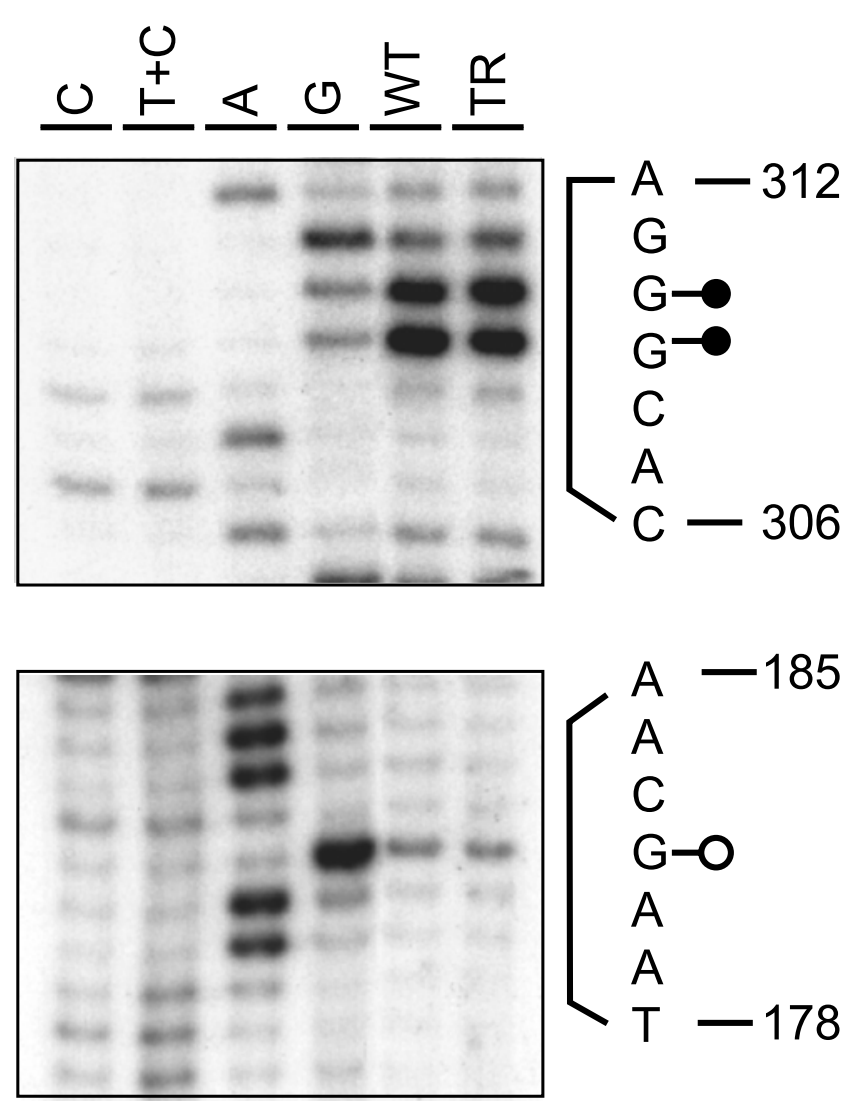

Figure 2. Protein-DNA interactions are revealed in Mod4 by an in vivo footprinting assay. P10 normal mice (WT) and adult trembler mice (TR) were pretreated with dimethylsulfate. DNA prepared from their sciatic nerves was compared with similarly treated purified DNA (sequencing reaction lane $C, T+C, A$, and $G$ ). Protection is detected on base 182, whereas bases 309 and 310 are hypersensitive.

in motif 15 (Figs. 1,2). These observations suggest that a protein complex binds to the most conserved Mod4 region, whereas the sequences surrounding Mod4 lack similar interactions with transcription factors. Consequently, DNA-protein interaction analysis and functional characterization focused on Mod4, guided by the location of phylogenetic footprints.

\section{MBP developmental programming is exposed by} species-specific Mod4 expression

To determine whether human and chicken Mod4 sequences are capable of productive interaction with mouse transcription factors, each was ligated to an hsp68-promoted LacZ reporter gene and investigated for expression in transgenic mice. To eliminate the variation associated with random integration at different insertion sites, constructs were docked, in single copy and predetermined orientation, in the $5^{\prime}$ flanking sequence of the HPRT locus (Bronson et al., 1996). Mod4 sequences from both species drove reporter gene expression in Schwann cells in mature mice (Fig. $3 A, B$ ). The human sequence conferred a typical postnatal expression phenotype initiating high-level expression at the commencement of myelin formation. In contrast, the chicken Mod4 reporter remained silent during preweaning development, when both the endogenous MBP locus and the human Mod4 reporter are highly expressed. Rather, expression initiated in the periweaning period when myelin formation in the PNS is approaching a mature state. Thus, Mod4 elements essential for expression during mammalian myelin formation are either nonexistent or too divergent in the chicken sequence to be recognized by mouse 
A
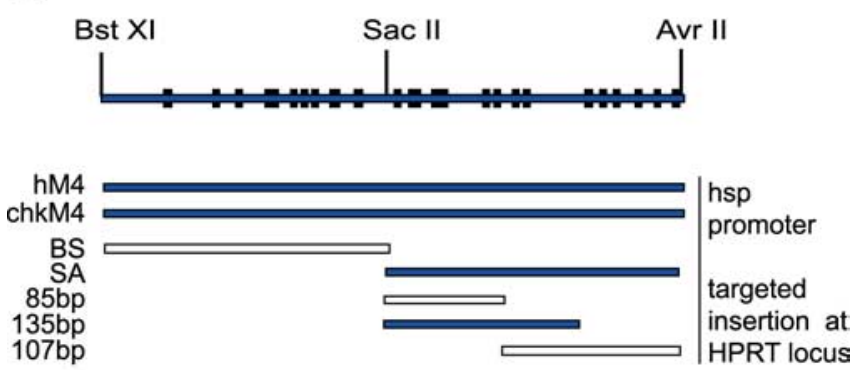

B

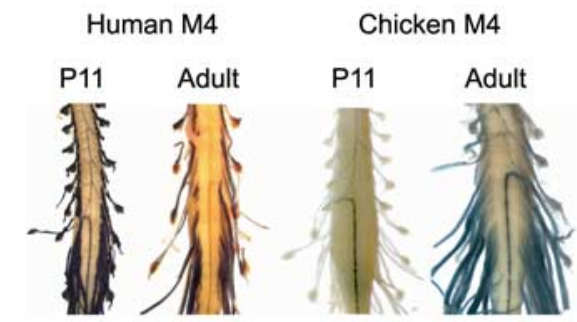

C

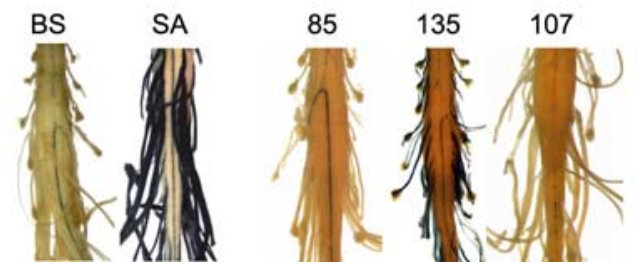

Figure 3. A, Distribution of conserved Mod4 motifs in sequences analyzed for in vivo function. Sequences driving Schwann cell expression are indicated in blue. $\boldsymbol{B}, \beta$-Galactosidase histochemistry was performed on whole-mount preparations of spinal cords (CNS) with attached spinal roots (PNS) to reveal Schwann cell-specific targeting. The human Mod4 construct expresses in spinal roots at P11 and in the adult, whereas the chicken Mod4 construct expresses in the spinal roots only in adults. C, The SA and 135 bp constructs are expressed in spinal roots but not in spinal cord oligodendrocytes. Note that the ventral spinal cord shown here demonstrates obvious labeling of the central artery; however, because this is typical of diverse reporter constructs docked at HPRT, it does not represent Mod4-specific enhancer activity.

factors. Additionally, the location of such essential elements must be limited to the polymorphisms existing between the chicken and human sequence (Fig. 1). The subsequent expression of the chicken Mod4 reporter in mature mice demonstrates that the MBP relevant transcription factor repertoire undergoes significant changes as Schwann cells progress from myelin elaboration to myelin maintenance.

\section{Mapping of the targeting sequence within Mod4}

To map the location of targeting elements within mouse Mod4, multiple Mod4 subdomains were analyzed individually (Fig. $3 A, C)$. The 5' 210 bp lying between BstXI and SacII (BS) containing conserved motifs M1-M9 failed to drive Schwann cell expression. In contrast, robust expression was conferred by the $3^{\prime}$ 213 bp lying between SacII and AvrII (SA) that contains motifs $10-22$. Expression of this construct is specific to the PNS, because no staining was observed in spinal cord white matter where oligodendrocytes elaborate myelin (Fig. $3 B, C$ ). Note the two nonoverlapping fragments of SA; 85 bp containing M10-M13 and 107 bp containing M14-M20 individually showed no activity. Thus, one or more elements within each fragment are needed to confer targeting activity. Because a 135 bp sequence containing M10-M16 drives Schwann cell expression, we conclude that at least one of the essential elements is located within the $50 \mathrm{bp}$ containing M14-M16.

\section{Subfragments of Mod4 confer responsiveness to axonal signaling}

In the rat, expression of many myelin-related genes follows the program of myelin acquisition, with peak expression realized in the third week ex utero (Stahl et al., 1990). We show here that MBP mRNA follows a similar program in the mouse (Fig. 4A). To determine whether the core enhancer sequences contained in the SA and 135 bp fragments are sufficient to confer this developmental expression program, the quantitative phenotypes realized from the respective reporter genes were evaluated throughout development. Both constructs yield postnatal expression phenotypes that track the major features of the endogenous MBP program; for SA, a sixfold increase occurs from P2 to P14, followed by a decrease to one-fourth the peak level by 3 months of age (Fig. $4 A$ ). However, peak expression occurs earlier for these constructs, which could be a result of different stabilities of lacZ and MBP mRNA or transcriptional differences related to the constructs or their insertion environment. One notable exception, observed for both the SA and 135 sequences, is expression in fetal nerves commencing at embryonic day 13.5 postcoitum (data not shown). Such precocious expression indicates that they lack one or more elements essential for normal silencing of MBP transcription in Schwann cell precursors.

High-level expression of genes encoding the major myelin proteins requires that Schwann cells achieve and maintain axonal contact. After axon transection, denervated Schwann cells in the distal nerve segment abruptly downregulate numerous myelinrelated genes such as MBP (LeBlanc and Poduslo, 1990). These are reexpressed only when contact with in-growing axons is reestablished. To determine whether the elements within the SA construct are sufficient to confer this injury/remyelination response, $\beta$-galactosidase activity was evaluated in sciatic nerves regenerating in response to crush injury (Fig. $4 \mathrm{~B}$ ). Consistent with maintained axonal responsiveness, 1 week after crush, $\beta$-galactosidase activity in the distal segment was reduced to onethird that expressed by uninjured contralateral nerves. When ingrowing axons reestablish contact with Schwann cells in the distal segment (approximately 2 weeks after crush), $\beta$-galactosidase levels were indistinguishable from control nerves, and this restored activity was maintained 4 weeks after injury, when axon regeneration and remyelination are essentially complete. The same axon responsive expression program was revealed for the $135 \mathrm{bp}$ construct (histochemical analysis) (data not shown). Thus, the expression of both the $213 \mathrm{bp} \mathrm{SA}$ and internal $135 \mathrm{bp}$ sequence parallel MBP expression in response to axonal signals.

Mod4 contains distinct targeting and enhancing subdomains As observed in both developing and remyelinating preparations, Schwann cell targeting and responsiveness to axon signals are conferred by elements located within the core 135 bp sequence. To evaluate the potential role played by motifs located outside this domain, constructs bearing additional motifs were analyzed for both qualitative and quantitative expression phenotypes. When compared with the $135 \mathrm{bp}$ sequence, SA extends for $78 \mathrm{bp}$ to include M17-M22, and it drives expression at threefold greater levels (Fig. 5). In the 5' half of Mod4, where M1-M9 are located, three constructs regulated by contiguous MBP $5^{\prime}$ flanking sequence, terminating at different sites within or $5^{\prime}$ of conserved Mod4 sequence, were analyzed. The sequence terminating at SacII includes motifs 10-22. The sequence terminating at 
-9.08 kb adds M5-M9 and demonstrates a marked increase in expression level during the preweaning period of myelin formation. Extension to $-9.5 \mathrm{~kb}$ adds M1-M4 and leads to an additional increase in expression levels in both juvenile and adult mice. Thus, one or all of the M5-M9 motifs confer regulatory information and recognize factor(s) that distinguish preweaning myelin elaboration from myelin maintenance in mature mice.

\section{Individual motifs in Mod4 subdomains are functional}

To assign function to specific elements, we performed both band shift assays using motif specific oligonucleotides and expression analysis with constructs bearing single-motif mutations. EMSAs were performed with probes representing eight conserved motifs in the SA sequence (Fig. $1 B)$. Although such putative elements could function in diverse cell types, we searched for those that could engage transcription factors expressed in Schwann cells by incubating the oligonucleotides with nuclear extracts prepared from P10 sciatic nerves. Consistent shifts were detected with five of the eight probes (M11, M12-5', M14, M16, and M20) (Fig. 6A, B). None were competed with oligonucleotides bearing 3-5 bp substitutions in the motif cores. Thus, some motifs appear to engage factors expressed in Schwann cells, whereas those that were not shifted may require cooperative binding not achievable on short oligonucleotides or engage factors expressed in different cell types.

Motifs M14 and M16 are located in the 50 bp shown to be essential for Schwann cell targeting, and M18 and M20 are the most conserved motifs present in the enhancing region of SA. Therefore, we focused our search for candidate transcription factor binding elements on these sites. Using rVISTA software (Loots et al., 2002), several candidates with known relevance to Schwann cell biology and myelination were revealed. M14 and M18 contain Sox family binding sites, although we were unable to confirm this function by supershift. M16 contains sites for AP1 and nuclear receptors RORA1 and ER (Desarnaud et al., 2000; Miskimins and Miskimins, 2001), whereas M20 is a consensus binding site for Krox-20.

To determine whether M20 can bind Krox-20, we used a Krox-20 antibody in a supershift analysis (Chavrier et al., 1988). With sciatic nerve extract, the M20 oligonucleotide yields a complex of three retarded bands, two of which are specifically competed by an excess of cold oligonucleotide. When the Krox-20 antibody is present in the reaction, it displaces the upper specific complex. As a control, the incubation was performed with Sp1 antibody, and only the nonspecific complex was displaced. As a further test of specificity, we expressed Krox-20 in bacteria and used bacterial extract for M20 band shift; a single complex formed, and it was specifically competed with a Krox control oligonucleotide. In addition, the sequence of M8, in the $5^{\prime}$ half of Mod4, is compatible with Krox-20 binding activity and consis- tent with this function, it also binds Krox-20 from sciatic nerve extracts or bacterial extracts (data not shown). We conclude that motifs M8 and M20 are able to bind Krox-20, and therefore, Krox-20 is a factor contributing to Mod4 enhancer activity in Schwann cells.

To test the in vivo function conferred through individual motifs, constructs were derived in which substitution mutations were introduced into the core of M14, M16, M18, and M20 motifs (Fig. 1), and their consequences on reporter gene expression phenotypes were analyzed in transgenic mice. When introduced in the context of the core 135 bp sequence, M14 and M16 mutations each affected expression; the M14 mutation entirely silenced expression throughout postnatal development, whereas the M16 mutation reduced expression to $20 \%$ of control levels in young animals and to $40 \%$ in adults (Fig. 6C). The large reduction of expression observed with both mutants suggests that a cooperative mechanism, involving both motifs, confers functionality to the $135 \mathrm{bp}$ subdomain.

M18 and M20 were similarly mutated but in the context of the longer SA sequence. Both mutations caused expression levels to decline to $\sim 50 \%$ of control values at most developmental stages. An exception is the M18 mutation at P4, where activity reached only $30 \%$ of the control value.

Because M14 and M18 share a common AACAA sequence [close to the Sox protein consensus AACAA(T/A)RG for Sox9 in Transfac], and both motifs play an essential role in enhancer function, they may bind a particularly critical factor. To deter- 
A

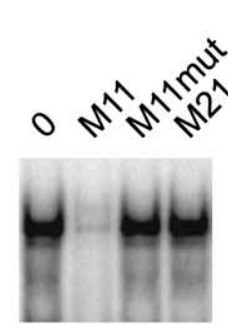

M11 $\hat{1}_{0}$

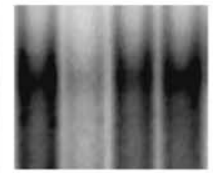

M12-5
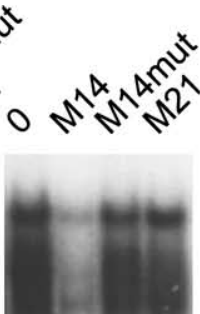

M14

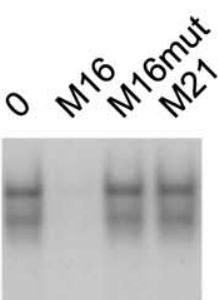

M16
B
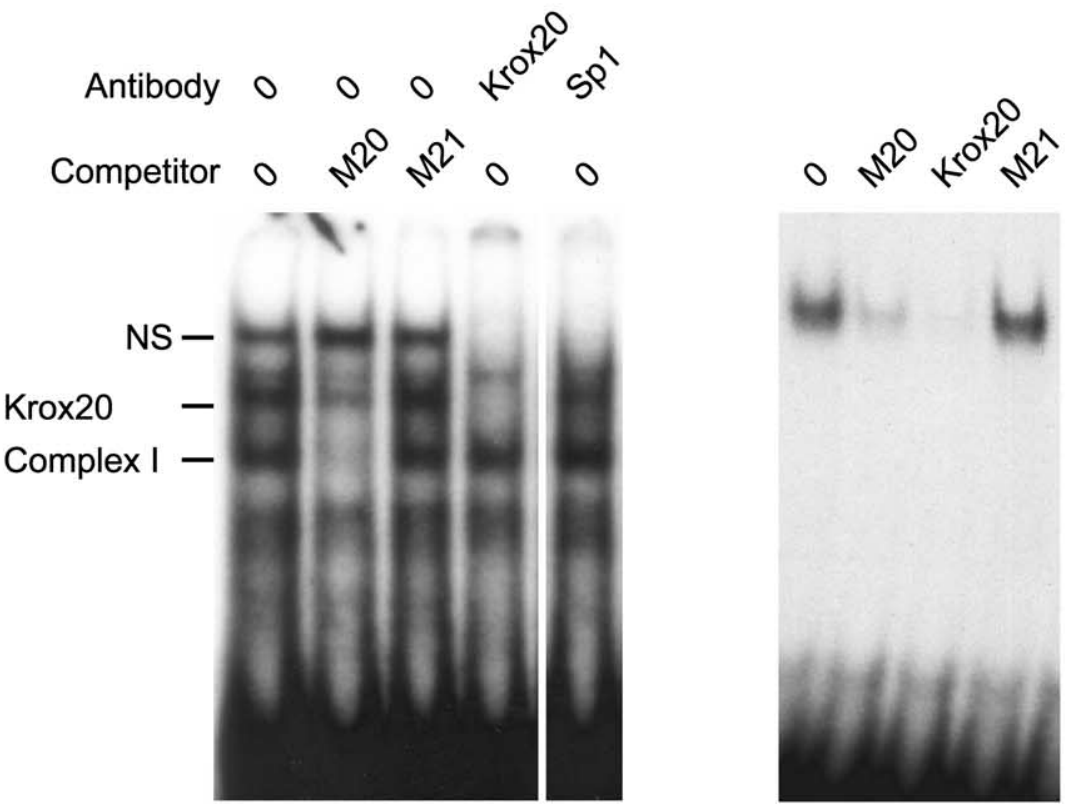

C
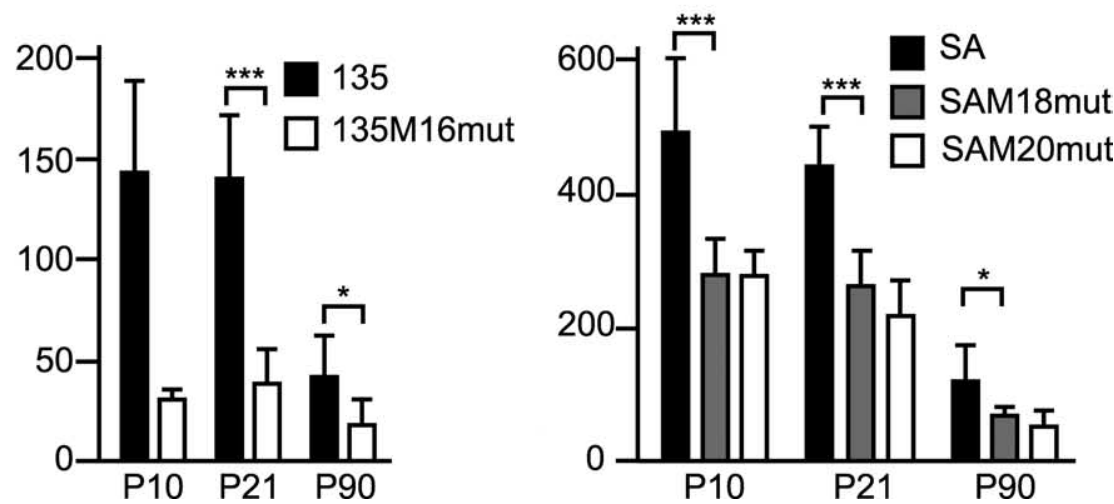

Figure 6. A, Interaction of motifs M11, M12-5', M14, and M16 detected by EMSA. Labeled oligonucleotides were incubated with sciatic nerve extracts from P10 mice. Competition was performed with the oligonucleotides indicated (top of each lane). Note that two specific complexes are formed with oligonucleotide M16. B, M20 binds Krox-20 from sciatic nerve extracts (left) or bacterially expressed Krox-20 (right). Competition was achieved with the indicated oligos. Characterization of Krox-20 in sciatic nerve extracts was done by supershift with Krox-20 antibody and Sp1 antibody as a control. C, $\beta$-Galactosidase activity in sciatic nerve samples from mice bearing control constructs (135 and SA) or mutated constructs (135M16mut, SAM18mut, and SAM20mut). The 135M14mut has no activity and is not represented. Means $\pm S D$. $t$ test results are indicated as ${ }^{*} p<0.05$ and ${ }^{* *} p<0.001 ; n \geq 5$.

mine whether this factor could play a similar role in the regulation of other Schwann cell expressed genes, we searched for this motif in those sequences shown previously to confer Schwann cell targeting in transgenic mice. As shown in supplemental Table
1 (available at www.jneurosci.org as supplemental material), the motif was present in multiple copies and conserved in the Oct6 Schwann cell enhancer, Krox-20 myelinating cell enhancer, PMP22 conserved region 1, and a conserved region lying in 6 $\mathrm{kb}$ of sequence upstream of the P0 gene. This observation suggests that the factor bound to the M14 motif is a widely used component of the mechanism regulating myelin gene expression in Schwann cells.

\section{Functional organization of Mod4}

The functional organization of $\operatorname{Mod} 4$ shares numerous features with the regulatory mechanism controlling the well characterized endo-16 locus of the sea urchin (Yuh et al., 1998). Specifically, enhancer function is conferred through a small number of targeting elements operating in concert with multiple additional elements modulating activity in response to physiological and developmental changes. When the different constructs investigated in the present study are aligned with the Mod 4 sequence conservation plot (Fig. 7), the elements necessary for Schwann cell specificity are found in two peaks of conservation at the $5^{\prime}$ and $3^{\prime}$ ends of the core $135 \mathrm{bp}$ targeting sequence. In combination, they are essential for the basic Schwann cell targeting function. In contrast, the two peaks of conservation flanking the $135 \mathrm{bp}$ targeting sequence contribute enhancing activities, some of which are restricted to defined stages in Schwann cell maturation.

\section{Discussion}

In this investigation, we show that Mod4 is composed of 22 conserved motifs. We provide evidence that most, if not all, contribute to Schwann cell enhancer activity with motif M14 playing an essential role in enhancer function while others modulate quantitative output.

M14 and M18 are putative Sox family binding elements. Because Sox proteins contain a high-mobility group domain known to bend DNA, Sox binding is expected to facilitate the cooperative binding of additional transcriptional activators leading to functional complexes (Bustin, 1999; Ellwood et al., 2000). Consistent with this role, Sox10 is known to modulate expression of myelin genes such as $\mathrm{P0}$ in the PNS (Peirano et al., 2000) and MBP in the CNS (Wei et al., 2004). Through interaction with M14, it may also be the critical factor required for the formation of the MBP Mod4 Schwann cell enhancer complex. Remarkably, the core M14 and M18 sequence was found, in multiple copies, in all known sequences capable of driving Schwann cell expression in transgenic mice. Thus, Sox proteins 
may play a fundamental role in the regulation of multiple genes expressed by differentiating Schwann cells. A similar analysis with the other Mod4 motifs should indicate how widely the factors and elements engaged in the enhancer activity are used in coordinating the overall myelination program.

In addition to the two putative Sox protein binding sites, Mod4 also contains two motifs that are able to bind Krox-20. Schwann cells arrest in a premyelinating state in Krox-20 knock-out mice, and a direct role for Krox-20 in the regulation of connexin-32 has been shown previously (Musso et al., 2001). Here, we show that Krox-20 plays a direct role in MBP regulation. However, in our 135 bp construct, no obvious binding site for Krox-20 was found, suggesting that Krox-20 is not crucial but rather, as seen for the periaxin gene (Parkinson et al., 2003), is used to amplify the activity of the enhancer. These combined results suggest that Krox-20 plays an important role in late myelin gene expression.

Binding site redundancy has been observed in multiple enhancers leading to a general model in which enhancers are thought to activate through multiple combinations of bound factors (Berman et al., 2002). We show here that such a redundancy exists within Mod4. By focusing our functional analysis on short, but still functional subsequences, we demonstrated large consequences of motif mutation and deletion. Whether consequences of the same magnitude would be elicited by the motif mutations introduced in the context of the entire module remains to be demonstrated.

Using in vivo functional analysis, we demonstrate that Mod4 enhancer activity requires simultaneous contributions from elements located in both targeting and enhancing subdomains. A more proximal MBP oligodendrocyte enhancer (Mod3) demonstrates a similar structure (N. Dionne, personal communication), suggesting a general model of enhancer structure/function in which targeting, once established, allows for fine-tuning of expression phenotypes through the lateral recruitment of enhancing elements. Evolutionary diversification of such lateral elements could accommodate species-specific regulatory requirements, and consistent with this hypothesis, divergence between mammalian and chicken Mod4 is more pronounced outside the targeting core. The extent to which this model is generally applicable will become evident as the identity and location of functional elements in additional tissue-specific enhancers become known.

A recent study (Taveggia et al., 2004) also used reporter constructs to characterize the MBP sequences important for expression in primary cultures of Schwann cells and oligodendrocytes. In such preparations, the Mod4 sequence, in the context of the $-9.0 \mathrm{~kb}$ MBP construct, was found to enhance activity in oligodendrocytes. In contrast, among the transgenic lines we have evaluated to date, no construct regulated by Mod4, or its derivatives, expresses in oligodendrocytes. However, we cannot exclude the possibility that Mod4 modulates the quantitative oligodendrocyte expression controlled by other MBP modules. Alternatively, Taveggia et al. (2004) point out that glial cells cultured in the absence of neurons may not provide a normal regulatory environment. Consistent with this limitation, we show here, in vivo, that Mod4 enhancer subdomains are highly responsive to axon signals.

The consequences of the experimental mutations introduced in this study suggest that naturally occurring variation within regulatory sequences could lead to significant gene deregulation (Knight, 2005). Mutations in conserved motifs could variously silence, or significantly downregulate, transcription at multiple developmental stages. The variable age of onset in numerous diseases, including myelinopathies, could in part be caused by variation affecting the stage-specific regulatory motifs of key genes.

Finally, this investigation shows the important role in vivo functional analysis can play in the investigation of mammalian regulatory mechanisms. Few techniques are capable of accurately revealing the expression phenotypes conferred by specific regulatory elements, and these are most widely applied to nonvertebrate models or in vitro preparations. The results of this investigation further demonstrate that the controlled construct docking strategy introduced by Bronson et al. (1996) can be applied as an effective strategy to reveal high-resolution qualitative and quantitative in vivo expression phenotypes. By supporting comprehensive access to temporal, spatial, and quantitative regulatory phenotypes, this robust in vivo approach emerges as an effective complement to both bioinformatics and molecular investigations on the structure and function of mammalian regulatory sequence.

\section{References}

Berman BP, Nibu Y, Pfeiffer BD, Tomancak P, Celniker SE, Levine M, Rubin GM, Eisen MB (2002) Exploiting transcription factor binding site clustering to identify cis-regulatory modules involved in pattern formation in the Drosophila genome. Proc Natl Acad Sci USA 99:757-762.

Bermingham Jr JR, Scherer SS, O'Connell S, Arroyo E, Kalla KA, Powell FL, Rosenfeld MG (1996) Tst-1/Oct-6/SCIP regulates a unique step in peripheral myelination and is required for normal respiration. Genes Dev 10:1751-1762.

Bondurand N, Girard M, Pingault V, Lemort N, Dubourg O, Goossens M (2001) Human Connexin 32, a gap junction protein altered in the $\mathrm{X}$-linked form of Charcot-Marie-Tooth disease, is directly regulated by the transcription factor SOX10. Hum Mol Genet 10:2783-2795.

Britsch S, Goerich DE, Riethmacher D, Peirano RI, Rossner M, Nave KA, Birchmeier C, Wegner M (2001) The transcription factor Sox10 is a key regulator of peripheral glial development. Genes Dev 15:66-78.

Bronson SK, Plaehn EG, Kluckman KD, Hagaman JR, Maeda N, Smithies O (1996) Single-copy transgenic mice with chosen-site integration. Proc Natl Acad Sci USA 93:9067-9072.

Bustin M (1999) Regulation of DNA-dependent activities by the functional 
motifs of the high-mobility-group chromosomal proteins. Mol Cell Biol 19:5237-5246.

Chavrier P, Zerial M, Lemaire P, Almendral J, Bravo R, Charnay P (1988) A gene encoding a protein with zinc fingers is activated during G0/G1 transition in cultured cells. EMBO J 7:29-35.

Desarnaud F, Bidichandani S, Patel PI, Baulieu EE, Schumacher M (2000) Glucocorticosteroids stimulate the activity of the promoters of peripheral myelin protein-22 and protein zero genes in Schwann cells. Brain Res 865:12-16.

Drouin R, Gao S, Holmquist G (1996) Agarose gel electrophoresis for DNA damage analysis. In: Technologies for detection of DNA damage and mutations (Pfeifer GP, ed), pp 37-43. New York: Plenum.

Drouin R, Therrrien J-P, Angers M, Ouellet S (2001) In vivo DNA analysis. In: Methods in molecular biology, Vol 148, DNA-protein interactions: principles and protocols, Ed 2 (Moss T, ed), pp 175-219. Totowa, NJ: Humana.

Ellwood KB, Yen YM, Johnson RC, Carey M (2000) Mechanism for specificity by HMG-1 in enhanceosome assembly. Mol Cell Biol 20: 4359-4370.

Farhadi HF, Lepage P, Forghani R, Friedman HC, Orfali W, Jasmin L, Miller W, Hudson TJ, Peterson AC (2003) A combinatorial network of evolutionarily conserved myelin basic protein regulatory sequences confers distinct glial-specific phenotypes. J Neurosci 23:10214-10223.

Feltri ML, D'Antonio M, Quattrini A, Numerato R, Arona M, Previtali S, Chiu SY, Messing A, Wrabetz L (1999) A novel P0 glycoprotein transgene activates expression of lacZ in myelin-forming Schwann cells. Eur J Neurosci 11:1577-1586.

Forghani R, Nesbitt J, Snipes J, Shooter EM, Peterson A (1999) Preparation of nuclear extracts from myelinating Schwann cells. J Neurosci Methods 89:129-132.

Forghani R, Garofalo L, Foran DR, Farhadi HF, Lepage P, Hudson TJ, Tretjakoff I, Valera P, Peterson A (2001) A distal upstream enhancer from the myelin basic protein gene regulates expression in myelin-forming Schwann cells. J Neurosci 21:3780-3787.

Ghislain J, Desmarquet-Trin-Dinh C, Jaegle M, Meijer D, Charnay P, Frain M (2002) Characterisation of cis-acting sequences reveals a biphasic, axondependent regulation of Krox20 during Schwann cell development. Development 129:155-166.

Knight JC (2005) Regulatory polymorphisms underlying complex disease traits. J Mol Med 83:97-109.

Kuhlbrodt K, Herbarth B, Sock E, Hermans-Borgmeyer I, Wegner M (1998) Sox10, a novel transcriptional modulator in glial cells. J Neurosci $18: 237-250$

LeBlanc AC, Poduslo JF (1990) Axonal modulation of myelin gene expression in the peripheral nerve. J Neurosci Res 26:317-326.
Loots GG, Ovcharenko I, Pachter L, Dubchak I, Rubin EM (2002) rVista for comparative sequence-based discovery of functional transcription factor binding sites. Genome Res 12:832-839.

Maier M, Castagner F, Berger P, Suter U (2003) Distinct elements of the peripheral myelin protein 22 (PMP22) promoter regulate expression in Schwann cells and sensory neurons. Mol Cell Neurosci 24:803-817.

Mandemakers W, Zwart R, Jaegle M, Walbeehm E, Visser P, Grosveld F, Meijer D (2000) A distal Schwann cell-specific enhancer mediates axonal regulation of the Oct-6 transcription factor during peripheral nerve development and regeneration. EMBO J 19:2992-3003.

Miskimins R, Miskimins WK (2001) A role for an AP-1-like site in the expression of the myelin basic protein gene during differentiation. Int $\mathrm{J}$ Dev Neurosci 19:85-91.

Musso M, Balestra P, Bellone E, Cassandrini D, Di Maria E, Doria LL, Grandis M, Mancardi GL, Schenone A, Levi G, Ajmar F, Mandich P (2001) The D355V mutation decreases EGR2 binding to an element within the Cx32 promoter. Neurobiol Dis 8:700-706.

Nardelli J, Gibson T, Charnay P (1992) Zinc finger-DNA recognition: analysis of base specificity by site-directed mutagenesis. Nucleic Acids Res 20:4137-4144.

Parkinson DB, Dickinson S, Bhaskaran A, Kinsella MT, Brophy PJ, Sherman DL, Sharghi-Namini S, Duran Alonso MB, Mirsky R, Jessen KR (2003) Regulation of the myelin gene periaxin provides evidence for Krox-20independent myelin-related signalling in Schwann cells. Mol Cell Neurosci 23:13-27.

Peirano RI, Goerich DE, Riethmacher D, Wegner M (2000) Protein zero gene expression is regulated by the glial transcription factor Sox10. Mol Cell Biol 20:3198-3209.

Stahl N, Harry J, Popko B (1990) Quantitative analysis of myelin protein gene expression during development in the rat sciatic nerve. Brain Res Mol Brain Res 8:209-212.

Taveggia C, Pizzagalli A, Fagiani E, Messing A, Feltri ML, Wrabetz L (2004) Characterization of a Schwann cell enhancer in the myelin basic protein gene. J Neurochem 91:813-824.

Topilko P, Schneider-Maunoury S, Levi G, Baron-Van Evercooren A, Chennoufi AB, Seitanidou T, Babinet C, Charnay P (1994) Krox-20 controls myelination in the peripheral nervous system. Nature 371:796-799.

Wei Q, Miskimins WK, Miskimins R (2004) Sox10 acts as a tissue-specific transcription factor enhancing activation of the myelin basic protein gene promoter by p27Kip1 and Sp1. J Neurosci Res 78:796-802.

Yuh CH, Bolouri H, Davidson EH (1998) Genomic cis-regulatory logic: experimental and computational analysis of a sea urchin gene. Science 279:1896-1902. 